Tohoku J. Exper. Med., 1961, 73, 398-415

\title{
Some Observations Concerning the End-Plate Potential
}

By

\author{
Yutaka Oomura and Tadao Tomita \\ Department of Physiology, Faculty of Medicine, \\ Kyushu University, Fukuoka
}

(Received for publication, November 20, 1960)

According to general knowledge, the process of neuromuscular transmission can be described by the following scheme: nerve impulse-acetylcholine (ACh)end-plate potential (e.p.p.)-muscle impulse-contraction. The e.p.p. has been analysed in detail by Fatt \& Katz (1951), who assumed that the active phase of the neuromuscular transmission is a brief impulsive event lasting only a few msec, and that the decline of the e.p.p. is determined by the electrical properties of the resting muscle membrane. Furthermore they proposed that the end-plate membrane would be converted momentarily by ACh into a short-circuit of the membrane potential of the muscle fiber, in other words, that the action of ACh would produce a relatively non-selective increase of ion flux, which drives the junctional membrane potential toward an equilibrium potential near zero (Fatt \& Katz, 1951), or -10 to $-20 \mathrm{mV}$ (Castillo \& Katz, 1954b).

The active phase, that is, the time course of change of a shunting resistance at the end-plate region of the muscle membrane can be measured by application of the so-called voltage-clamp method (Hodgkin, Huxley \& Katz, 1952; Tasaki \& Hagiwara, 1957; Hagiwara \& Tasaki, 1958) and was actually demonstrated by Takeuchi \& Takeuchi (1958, 1959) and Oomura \& Tomita (1958).

The equilibrium potential for the e.p.p. which would be expected from the short-circuit theory and produced by the non-selective ion flux at the membrane can be shifted by changing the ionic composition of the surrounding fluid. It was found by the authors that the direction of the e.p.p. was reversed due to shift of the equilibrium potential by replacement of Ringer fluid with sodium--lack sucrose solution.

In the present paper, experiments are described in which the behavior of the end-plate region was studied using the voltage-clamp method and various experimental solutions. It was hoped that these experiments would produce additional knowledge on the production of the e.p.p. and of the reversed e.p.p. which has a great similarity to the inhibitory synaptic potential. 


\section{METHODS}

Sartorius frog muscles (Rana nigromaculata) were removed with a $3-4 \mathrm{~cm}$ nerve attached and put into a lucite-chamber filled with saline. The nerve was lifted out of the bath and kept in fixed position on the stimulating electrodes; shocks of supramaximal strength were applied. The muscle was immobilized by application of $1-2 \times 10^{-6} \mathrm{~g} / \mathrm{ml}$ d-tubocurarine chloride (Abbott).

Two microelectrodes, of the Ling \& Gerard type (1949), were inserted into the end-plate regions of the muscle, one for passing current through the membrane and the other for recording potential (Fig. 1). Superficial fibers of the muscle were explored with the recording electrode until the e.p.p. was obtained at a focal point in response to nerve stimulation. The current electrode was then inserted, usually within $50 \mu$ of the recording electrode. The microelectrode had a resistance usually of from 15 to $30 \mathrm{M} \Omega$.

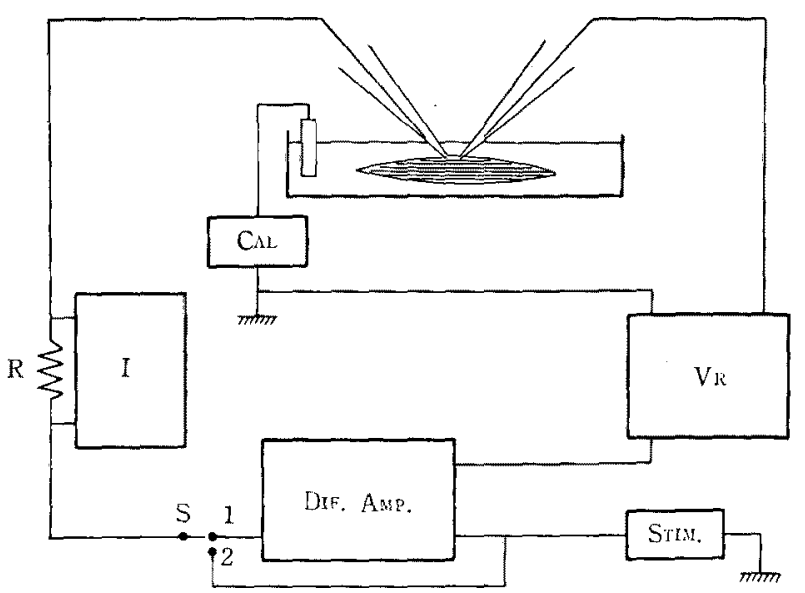

Fig. 1. Arrangement for measuring current and potential across the end-plate region of the muscle membrane with two intracellular microelectrodes. $V_{\mathrm{R}}$ : preamplifier, $\mathrm{I}$ : differential d.c. amplifier with input cathode followers for current recording, $\mathrm{R}: 200 \mathrm{~K} \Omega$, Cal. : calibrator, Stim. : square pulse generator, Dif. Amp. : differential input amplifier (modified from Tektronix type 122) for voltage-clamp experiment, $\mathrm{S}$ : switch, positioned at 1 for voltage-clamp experiment, at 2 for applying square pulse current to the muscle fiber.

The first stage of a balanced d.c. preamplifier designed by the authors was cathode follower type of low grid current $\left(2 \times 10^{-12} \mathrm{~A}\right)$, and a positive feed-back voltage was applied at the input to correct for the loss in high frequency response. This results in good frequency response (d.c. to $100 \mathrm{KC}$ ) and a gain of 20 times in amplification. For the voltage-clamp experiment, a negative feed-back amplifier 
system (a differential amplifier of Tektronix type 122 with a gain of approximately 1000 times) was connected between the two electrodes as shown in Fig. 1 so as to keep the membrane potential at a desired level (switch $\mathbf{S}$ was positioned at 1). When switch $\mathrm{S}$ was positioned at 2 , the feed-back circuit was opened and the polarizing current was fed into the current electrode from a pulse generator. The current was recorded at $\mathrm{R}$ (usually $200 \mathrm{~K} \Omega$ ) with a balanced d. c. amplifier and sometimes at a low resistance $(5 \mathrm{~K} \Omega$ ) connected between the indifferent electrode and the ground.

The bath solution was usually either Ringer (composition: $112 \mathrm{mM}-\mathrm{NaCl}$, $2.0 \mathrm{mM}-\mathrm{KCl}, 1.8 \mathrm{mM}-\mathrm{CaCl}_{2}, 2.4 \mathrm{mM}-\mathrm{NaHCO}_{3}$ ) or sucrose-Ringer $(\mathrm{NaCl}$ being replaced by $212 \mathrm{mM}$-sucrose without $\mathrm{NaHCO}_{3}$, the other components being unchanged), or saline mixtures in which $\mathrm{Cl}$ ions in Ringer solution were replaced fully by $\mathrm{NO}_{3}$ or $\mathrm{SO}_{4}$ or partly by $\mathrm{I}$ ions. Most experiments were carried out at room temperature $10-15^{\circ} \mathrm{C}$ in winter and $25-27^{\circ} \mathrm{C}$ in summer.

\section{RESULTS}

A. Relation between the e.p.p. and the end-plate current (e.p.c.) under the voltage-clamp condition

When square pulses of outward or inward current were sent into the membrane through the current electrode inserted in the end-plate region close to the recording electrode, the membrane potentials shifted towards either the depolarization or hyperpolarization and the amplitude of the e.p.p.'s was altered. As shown in Fig. 4 and Fig. 6 not only the relationship between the membrane potential and the current flowing through the membrane, but also the relationship between the e.p.p. size and the current were almost linear. This indicates that the applied current can flow uniformly within a limited area of the end-plate region, and that one can apply the voltage-clamp technique to a limited area of the end-plate region.

To keep the membrane potential at the resting level during the neuromuscular transmission, the current must be passed through the end-plate membrane by the negative feed-back system. This current is henceforth called the end-plate current, e.p.c. The e.p.c. is, in other words, the current sent into the membrane through the electrode inserted in the end-plate region during the transmission when the membrane potential is kept at the resting level by means of the voltageclamp method.

Fig. 2A shows the e.p.p. obtained from the curarized muscle without the feed-back system operating and Fig. 2B (upper tracing) shows the e.p.c. using the feed-back system. There were characteristic differences in the time course between the e.p.p. and the e.p.c.; the former had a half rising and half fall-, ing time of $0.78 \mathrm{msec}$ and $9.5 \mathrm{msec}$ respectively, whereas the latter had half 

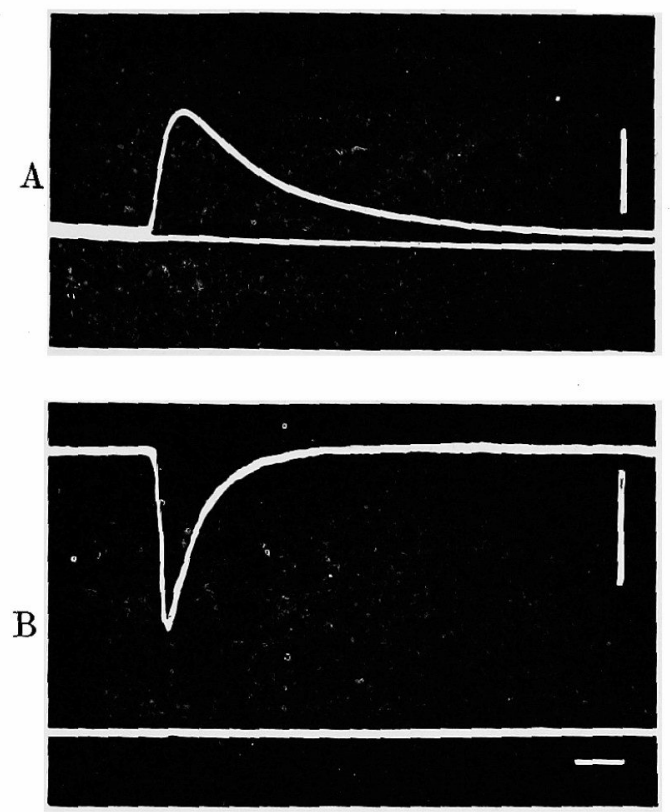

Fig. 2. A : e.p.p. at the focal point in curarized muscle. B : e.p.c. (upper beam) at the same region as $A$ when the membrane potential is kept at the resting level (lower oscilloscope trace). Temperature $15^{\circ} \mathrm{C}$. Calibrations: for voltage $10 \mathrm{mV}$, for current $4 \times 10^{-8} \mathrm{~A}$. Time : $5 \mathrm{msec}$. Resting potential, $85 \mathrm{mV}$.

rising and half falling time of $0.45 \mathrm{msec}$ and $3.2 \mathrm{msec}$. The e.p.p. and the e.p.c. were observed rising to a peak of $13 \mathrm{mV}$ in $2.7 \mathrm{msec}$ and $5.7 \times 10^{-8} \mathrm{~A}$ in $1.3 \mathrm{msec}$ respectively. As already shown by Fatt \& Katz (1951) a maximum of the endplate charge which may indicate a peak of the active phase was reached at $1.5-2$ msec, and the hump of the characteristic feature of the muscle action potential, recorded at the end-plate region by indirect stimulation was reached within 1.3-2 msec. The rising time to the peak of the e.p.c. was almost comparable to the two values indicated by Fatt \& Katz (1951).

The rising and falling curve of the e.p.c. can be expressed by the following equations :

$$
A \frac{t}{T} \quad(t=0 \text { to } T) \ldots \ldots \ldots \ldots \ldots \ldots \ldots \ldots \ldots \ldots \ldots
$$

and

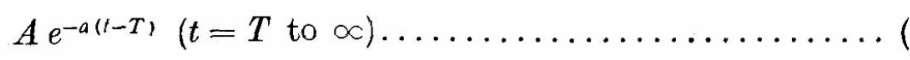

respectively. $A$ is peak value, $T$ is rising time and $a$ is a constant determined from the curve of the falling phase. From the equations it is assumed that the transmitter substance liberated from the nerve ending reached to receptor proteins by means of simple diffusion and were destoryed by the enzymatic action. 
The time course of reaction of the transmitter substance with the end-plate was studied by application of anticholinesterases or of sodium deficient solution by several investigators (Eccles, Katz \& Kuffler, 1942 ; Katz, 1948 : Eccles \& MacFarlane, 1949 ; Fatt \& Katz, 1951 ; Castillo \& Katz, 1955a). It was of interest to compare the time course of the e.p.p. with that of the e.p.c. in various amplitudes under the influence of various concentrations of curarine. The reason is as follows : If it is assumed that th? e.p.p. may be produced by synchronous manner of the transmitter from the so-called quantum units (Castillo \& Katz, 1954a) and that each transmitter may react in the same time course, then the rising and falling time of the e.p.c. in various amplitudes would almost be the same.

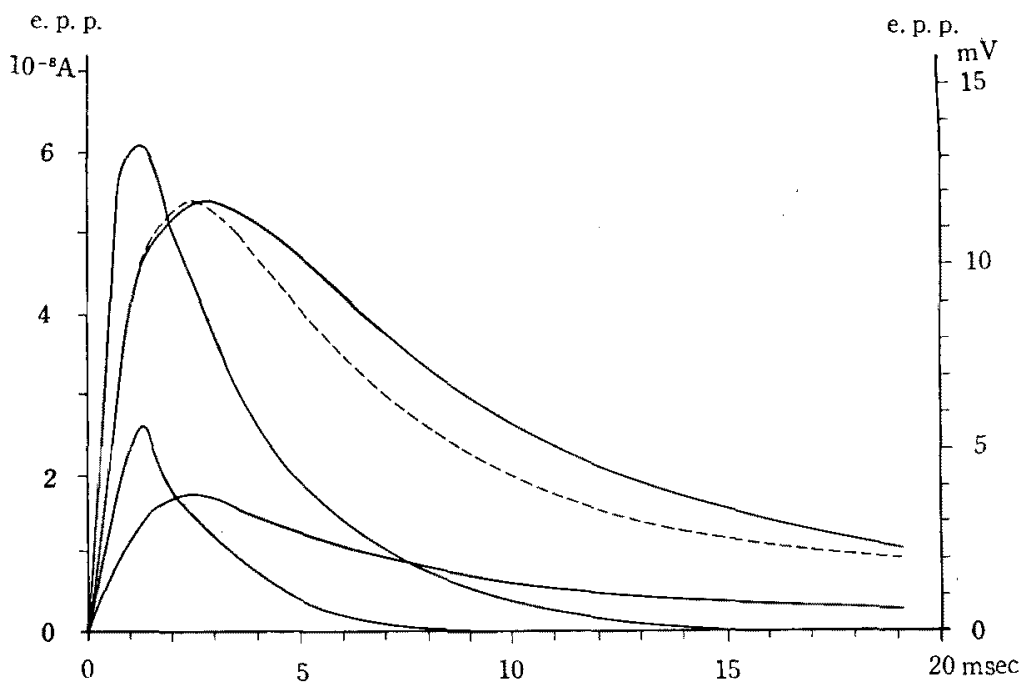

Fig. 3. Relationship between the e.p.p. (right) and the e.p.c. (left) in the curarized muscle. The magnitude of the e.p.p. was reduced by further application of curarine. The smaller the e.p.p.'s become, the rising and falling times to one-half become shorter and to make clear the time course between large and small e.p.p.'s, the ordinate is scaled to the same maximum as shown by dotted line. The e.p.c.'s have the same rising time to reach their peaks.

Fig. 3 shows a typical example of these experiments. The rising times to the peak in various intensities of the e.p.c. are almost the same, but in the falling phase the smaller the e.p.c.'s become, the half falling time becomes slightly shorter. (It was assumed that the time constant of the muscle membrane was not affected and a fixed amount of the transmitter was liberated during the experimental condition.) To make clear the time course between large and small e.p.p.'s the ordinates have been scaled to the same maximum as shown by dotted lines in e.p.p.'s. From these experimental results, it became clear that the above assumption is the case. The relationship between the magnitude of the e.p.p.'s and the intensity of 
the e.p.c.'s is shown in Fig. 7. Under usual experimental conditions the larger the amplitude of the e.p.p's, the intensity of the e.p.c.'s became larger and their relation was almost linear. However, for the larger e.p.p. the magnitude of the e.p.c. should become much larger, since the e.p.c. should be infinity (shunting resistance becomes zero) when the e.p.p. reaches the zero membrane potential.

When the membrane potentials under the clamped condition were shifted at various constant levels for $200 \mathrm{msec}$ and indirect stimulations were applied, the relationships between the membrane currents and the e.p.c.'s were obtained. When the membrane potential was shifted to hyperpolarization, an inward directed current appeared, preceded by a short capacitative surge, and it soon reached a final steady value (at a certain depolarized level, the current directed oppositely). The relationship between the steady currents and the shifted membrane voltages throughout depalarization and hyperpolarization was almost linear in many cases, and the calculated value of the effective resistance of the resting membrane from this relation was $200-600 \mathrm{~K} \Omega$. The amplitude of the e.p.c. in the hyperpolarization became larger compared with that at the resting level (smaller in depolarization). The peak amplitudes of the e.p.c. could be plotted against various clamped voltages.

A straight line connecting the plotted points could be drawn in many cases and crossed the abscissa where the membrane potential was -10 to $-30 \mathrm{mV}$. This may indicate that the equilibrium potential for the e.p.p. was number of $\mathrm{mV}$ under zero (Castillo \& Katz, 1954b), and that the minimum value of the shunting resistance calculated from the tangent of this relation was $600 \mathrm{~K} \Omega$ to $2 \mathrm{M} \Omega$ under curarized condition.

\section{B. Reversed e.p.p. due to the application of sodium deficient Ringer solution}

It was found by the authors that the amplitude of the e.p.p. became smaller but did not disappear and the direction of the e.p.p. was reversed when Ringer solution was replaced with sucrose-Ringer solution, and soon returned to normal by application of Ringer solution. This phenomenon did not happen in all muscles, but tended to take place in muscles with low resting potentials. One factor for this irregularity may be the great variability of $\mathrm{Na}$ content in the muscle (Simon, Shaw, Bennett \& Muller, 1957). The time course of the reversed e.p.p. was not so altered. Nerve conduction was not affected by Na-lack solution, possibly because the perineural barriers might protect the nerve from the effect of $\mathrm{Na}$-lack.

The magnitude of the reversed e.p.p. was increased by depolarization of the membrane potential and decreased by hyperpolarization, and this was directly opposite to cases of the normal e.p.p. (Fig. 4A). The relationships between membrane potentials and the reversed e.p.p. are shown in Fig. 4B. The rectification of the membrane by out-ward currents disappeared in this case and the 

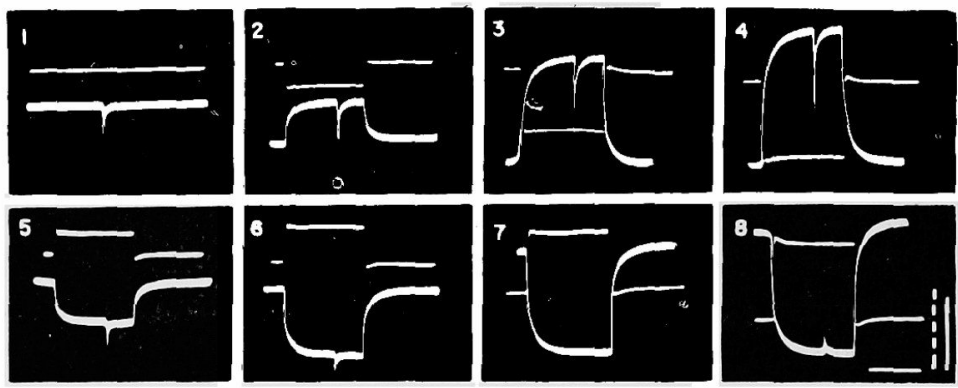

Fig. 4. A : reversed e.p.p. in Na-lack sucrose Ringer solution. 1, at the resting potential; $2-4$, at various membrane potential levels at depolarization; 5-8 at hyperpolarization. Upper beams show applied currents (in 7 and 8, lower beam) and lower beams membrane potentials (in 7 and 8, upper beams). The magnitudes of the reversed e.p.p. become larger at depolarization and smaller at hyperpolarization. Calibrations : solid bar, $10 \mathrm{mV}$; dotted line, $5 \times 10^{-8} \mathrm{~A}$; horizontal bar, $100 \mathrm{msec}$.

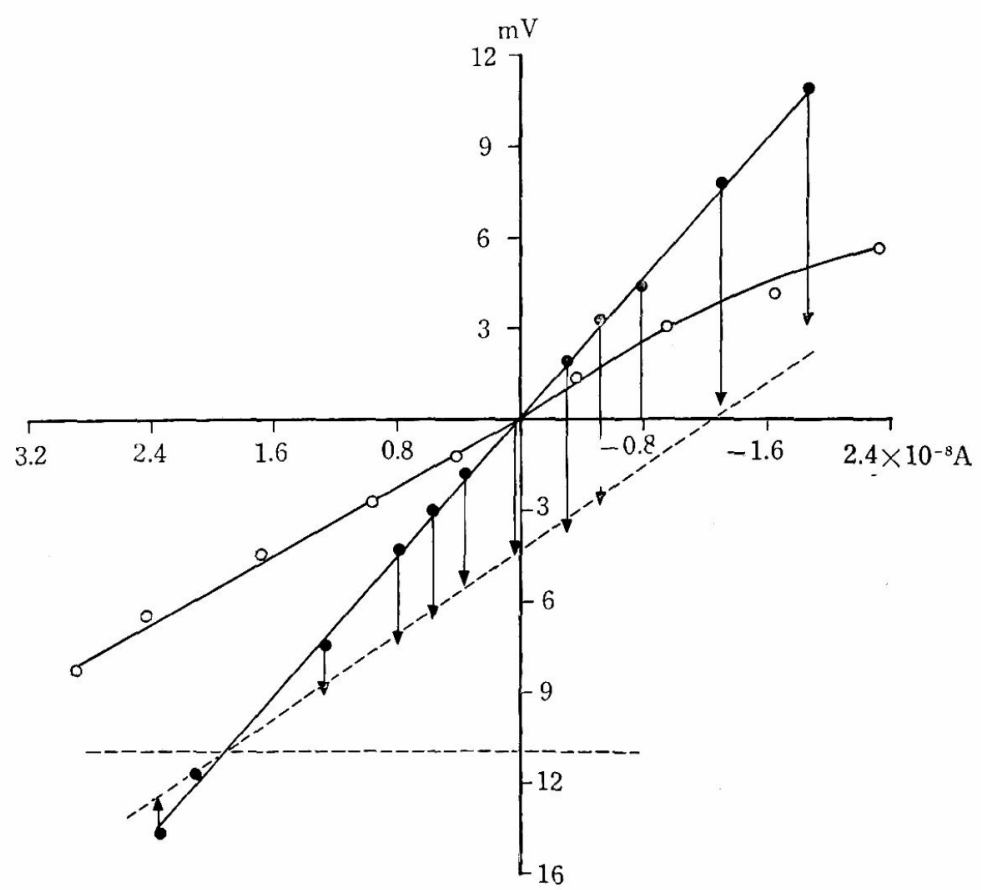

Fig. 4. B : relationship between the membrane potential and the reversed e.p.p.'s. Ordinate is the potential shift ( $O$ indicates the resting potential) by the applied current plotted on abscissa. Inward current is negative. Empty circles in Ringer solution and filled ones in Na-lack sucrose Ringer solution. Perpendicular bars show the magnitude of the reversed e.p.p.'s at various membrane potential. The reversed e.p.p. is again reversed at dotted line which indicates the equilibrium potential for the reversed e.p.p. In this case the equilibrium potential is located at hyperpolarized level by- $11 \mathrm{mV}$ from the resting potential. 
effective resting membrane resistance increased by about double in sucrose-Ringer solution.

The resting potential was usually lowered by 10 to $20 \mathrm{mV}$. Magnitudes of the reversed e.p.p's are indicated by lengths of perpendicular bars. When inward current intensity was further increased (at dotted line) the reversed e.p.p. was again reversed so that the direction of the e.p.p. become normal. As already mentioned, the shunting resistance of the membrane potential produced by the neuromuscular transmission means an increase in the non-selective ion fux at the end-plate region, thus in Na-free solution the equilibrium potential should shift to somewhere near the resting potential (Castillo \& Katz, 1955b). In this case the amplitude of the reversed e.p.p. became zero at $-11 \mathrm{mV}$ from the resting potential. That is, the equilibrium potential was located by $-11 \mathrm{mV}$ from the resting potential at hyperpolarized level. In other experiments of $\mathrm{Na}$-lack $\mathrm{NO}_{3}$ or $\mathrm{Na}$-lack $\mathrm{SO}_{4}$ solution, the equilibrium potential came near the resting potential (see Fig. 10). Thus the reversed e.p.p. could be also obtained in $\mathrm{Na}^{-1 a c k ~} \mathrm{SO}_{4}$ solution.

When the membrane potential was clamped at the resting level in Na-lack Ringer solution, the reversed e.p.c. was recorded.

C. Effects of nitrate, sulphate and other anions on the e.p.p. and the e.p.c.

As mentioned above, sucrose-Ringer solution affected the end-plate region. In connection with this, influences of the various anions substituted for $\mathrm{Cl}$ ions of Ringer solution were observed on the end-plate region. These anions had a considerable effect on increasing the mechanical response of the muscle to stimulation (Kahn \& Sandow, 1950 : Hill \& Macpherson, 1954; Ritchie, 1954), and on increasing the time constant and the resistance of muscle membrane (Padsha, 1957: Hutter \& Padsha, 1959). On the other hand, these anions had little effect on cation movement in resting muscles (Edwards, Harris \& Nishie, 1957), but slowed $\mathrm{Cl}$ ion movement (Harris, 1958). The resting potential measured was almost insensitive to the nature of the external anion as reported by other investigators (Harris \& Martins-Ferreira, 1955 ; Lubin, 1957 ; Hodgkin \& Horowicz, 1959).

When $\mathrm{Cl}$ ions in Ringer solution were replaced partly or fully with $\mathrm{NO}_{3}$ or $\mathrm{SO}_{4}$ ions the membrane resistance measured at the resting state increased as shown in Fig. 5. There was a linear relationship between the resistance increase and the concentration of the anions. In this figure the resistance in $\mathrm{SO}_{4}$ solution does not increase as compared with that in $\mathrm{NO}_{3}$ solution, but in other cases it can be said that the resistance increased by about twice in both $\mathrm{NO}_{3}$ and $\mathrm{SO}_{4}$ cases by a full replacement of $\mathrm{Cl}$ ions.

The anion such as $\mathrm{NO}_{3}, \mathrm{SO}_{4}$ and $\mathrm{I}$ had a decurarizing action on the end-plate region, i.e., the curarized muscle moved by nerve stimulation. The magnitude of 


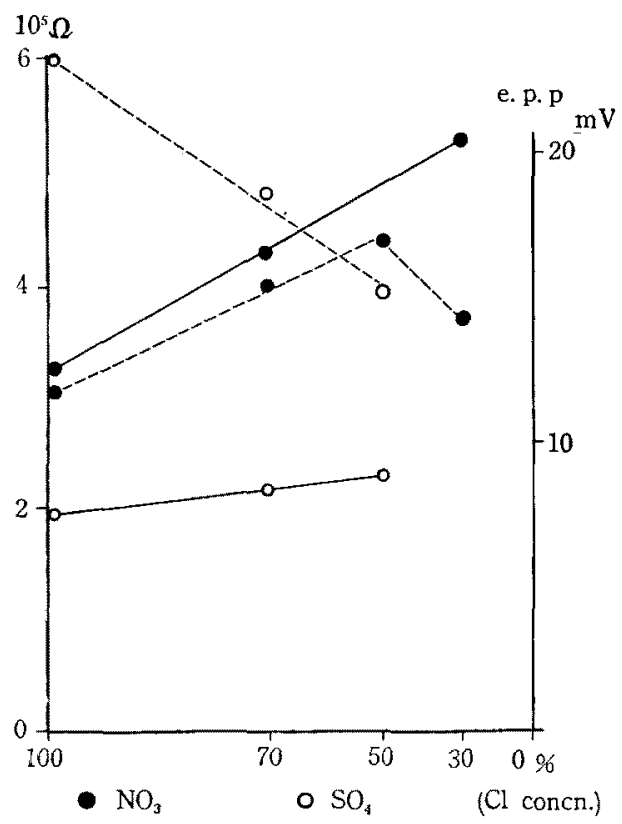

Fig. 5. Membrane resistance (left ordinate and solid line) and magnitude of the e.p.p. (right ordinate and dotted line) plotted against $\mathrm{Cl}$ ions concentration. $\mathrm{Cl}$ ions replaced by $\mathrm{NO}_{3}$ or $\mathrm{SO}_{4}$ ions. Membrane resistance becomes larger with the decrease of $\mathrm{Cl}$ ions concentration and magnitude of the e.p.p. becomes larger with the increase of $\mathrm{NO}_{3}$ ions and then in many cases decreases at higher concentration. In $\mathrm{SO}_{4}$ solution the magnitude of the e.p.p. becomes smaller.

the e.p.p. in $\mathrm{NO}_{3}$ solution became larger with the increase of concentration of $\mathrm{NO}_{3}$ and in many cases decreased at a very higher concentration of $\mathrm{NO}_{3}$, while the sensitivity for applied $\mathrm{ACh}$ on the end-plate region remained unaltered. This decrease was not explicable but one factor might be injury of the membrane caused by movement of the muscle. On the other hand the relationship between the magnitude of the e.p.p. and the concentration became opposite in $\mathrm{SO}_{4}$. These matters are summarized also in Fig. 5. The relationships between the magnitude of the e.p.p.'s and the membrane potentials are plotted in Fig. 6. The equilibrium potentials for the e.p.p. were not shifted so much in $\mathrm{NO}_{3}$ solution, and were about $-20 \mathrm{mV}$ of the membrane potential. When sucrose- $\mathrm{NO}_{3}$ solution was applied to the muscle the equilibrium potential was shifted toward the resting potential which was lowered by about $20 \mathrm{mV}$ from the value when $\mathrm{NO}_{3}$ solution was used. This was expected from the results of $\mathrm{Na}$-lack experiment.

In $\mathrm{NO}_{3}$ and $\mathrm{SO}_{4}$ solutions, the membrane resistance in the resting state increased. If one can assume that the time course of temporal change of the 


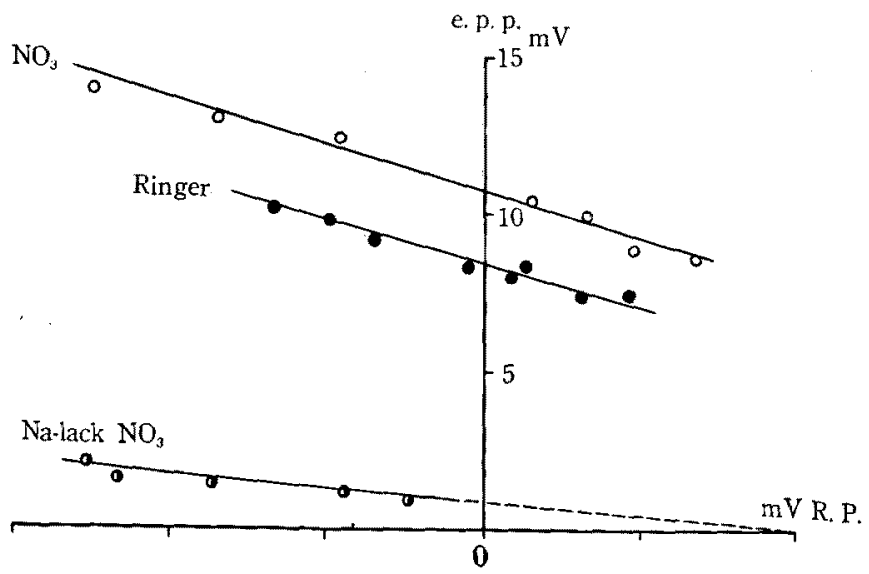

Fig. 6. Relationship between the magnitude of the e.p.p. and the membrane potential. Zero indicates the resting potential level (in $\mathrm{NO}_{3}$ the resting potential $-80 \mathrm{mV}$ ). Straight lines on the peak amplitude of the e.p.p.'s cross the abscissa in Ringer and $\mathrm{Cl}$ deficient solutions at the about same values of the membrane potential. The equilibrium potential for the e.p.p. shifted to depolarization by $+10 \mathrm{mV}$ from the resting potential in sucrose (the new resting potential in this solution (about $20 \mathrm{mV}$ reduced) is indicated also at zero).

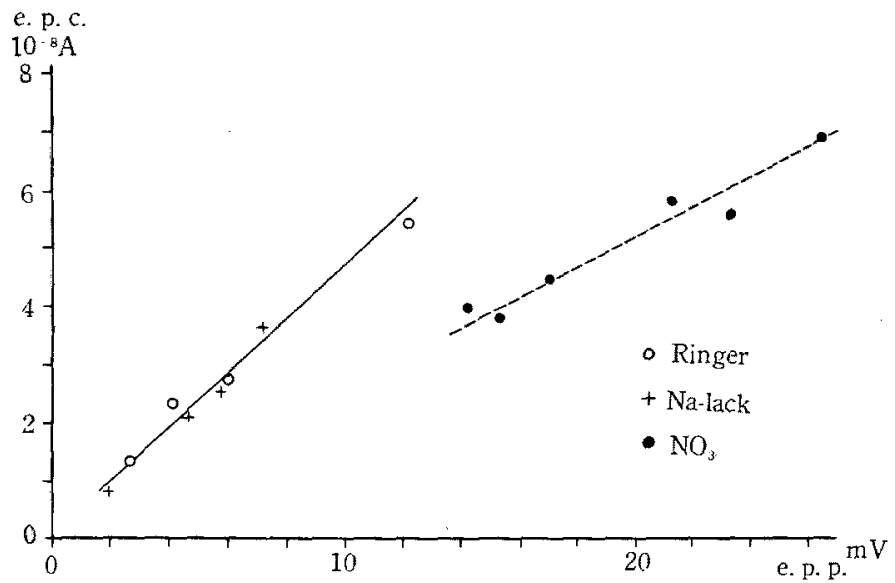

Fig. 7. Relationship between the magnitude of e.p.p.'s and intensity of the e.p.c.'s in Ringer, sucrose-Ringer and $\mathrm{NO}_{3}$ solution in the same fiber. Magnitudes of the e.p.p.'s are altered by rate of concentration of sucrose and $\mathrm{NO}_{3}$ in the solution. The magnitude of the e.p.p. in $\mathrm{NO}_{3}$ is much larger compared with that in Ringer solution at the same intensity of the e.p.e. Thus the enlargement of the magnitude of the e.p.p. in $\mathrm{NO}_{3}$ solution is due to the increase of the membrane resistance though the changing rate of the shunting resistance is not altered. 
shunting resistance produced by the transmitter action would not alter in both the normal and Cl-lack solutions, the magnitude of the e.p.p. should be expected to become larger in $\mathrm{NO}_{3}$ solution. The assumption can be proved when the intensities of the e.p.c.'s are measured in both $\mathrm{NO}_{3}$ and Ringer solutions. The relationships between the magnitude of the e.p.p.'s and intensity of the e.p.c.'s in Ringer, sucrose-Ringer and $\mathrm{NO}_{3}$ solutions are plotted in Fig. 7. Magnitudes of the e.p.p.'s were altered by the rate of the concentrations of sucrose and $\mathrm{NO}_{3}$ in the solution. It is quite clear from the figure that the magnitude of the e.p.p. in $\mathrm{NO}_{3}$ solution is much larger compared with that in Ringer solution at the same intensity of the e.p.c. Thus it can be said that the enlargement of the e.p.p. in $\mathrm{NO}_{3}$ solution was due to the increase of the membrane resistance though the rate of change of the shunting resistance was not altered.

In $\mathrm{SO}_{4}$ solution, as mentioned above, the magnitude of the e.p.p. became rather small in spite of the increase of the membrane resistance. $\mathrm{SO}_{4}$ ions have a strong precipitating action on $\mathrm{Ca}$ ions. The movement of the muscle fiber by an indirect stimulation was rather violent in $\mathrm{SO}_{4}$ solution. These two factors might tend to make the e.p.p. small, and further $\mathrm{SO}_{4}$ ions might have a specific action on the e.p.p. production.

\section{DISCUSSION}

Because the membrane potential is kept at the resting level by means of the voltage-clamp technique, the current produced by the neuromuscular transmission flows through only the shunting resistance in the end-plate region. If the current would flow through the membrane resistance or capacitance, the membrane

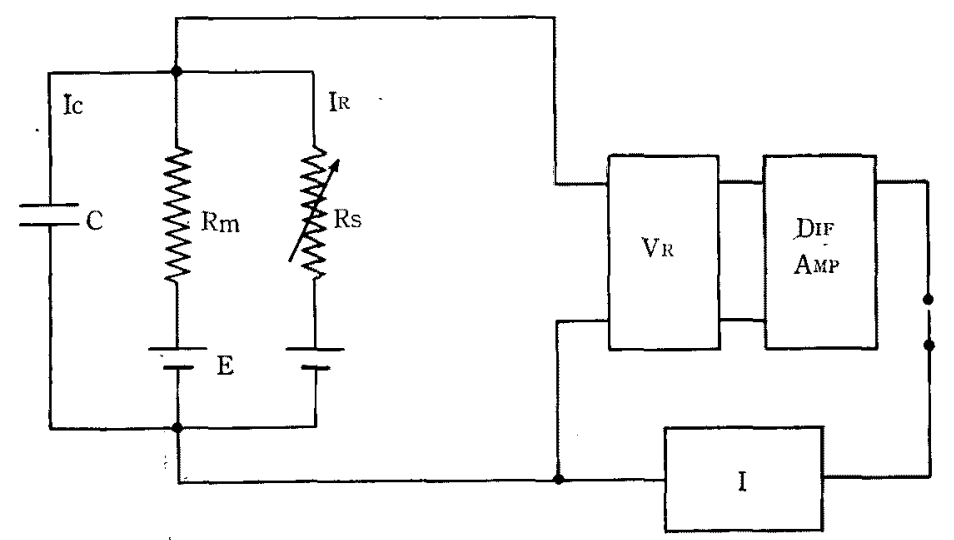

Fig. 8. A : electrical equivalent circuit diagram representing the end-plate membrane and circuit under the voltage-clamp condition. $\mathrm{E}$ : resting potential $(80 \mathrm{mV}), \mathrm{Rm}$ : resistance across the resting membrane $(200 \mathrm{~K} \Omega), \mathrm{C}:$ membrane capacitance $(0.1 \mu \mathrm{F}), \mathrm{Rm} \times \mathrm{C}$ is $20 \mathrm{msec}, \mathrm{Rs}$ : shunting resistance (changed from the infinity to a certain extent). 


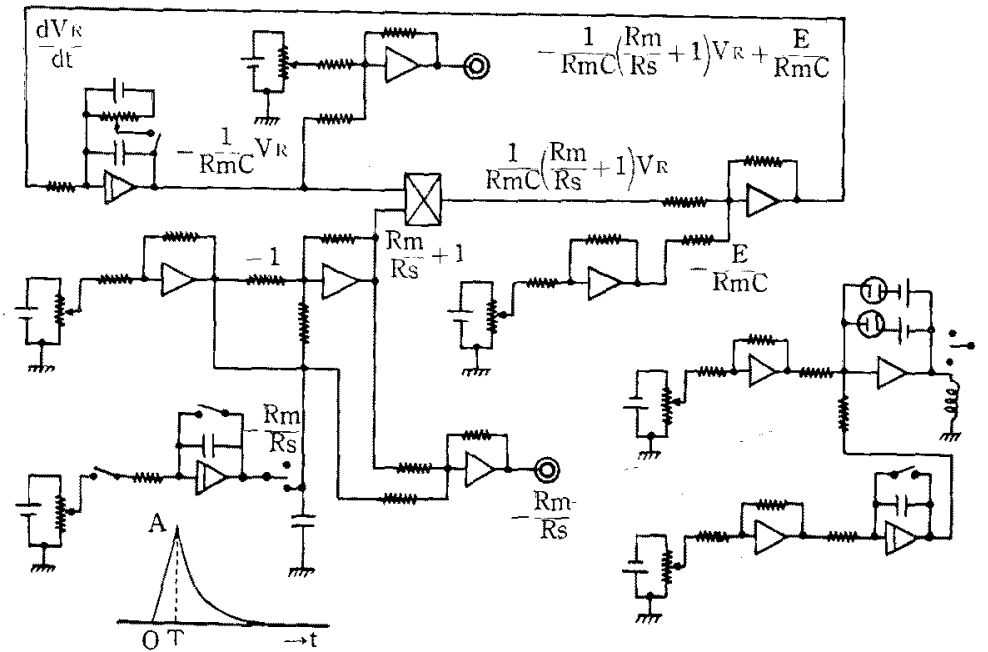

Fig. 8. B : circuit diagram of an analog computor for calculation of the differential equation (6). Inserted figure shows change of $R s$ with the time course as same as that of the e.p.e.

potential would shift from the resting level. If the equilibrium potential is zero, and the time course of the e.p.c. expresses change in the shunting resistance, it is clear that the product of the intensity of the e.p.c. multiplied by the value of the shunting resistance will be the value of the resting potential. In order to prove the above conclusion, it is necessary to determine whether changes in the membrane potential similar to the e.p.p. can be produced in the membrane by using the same e.p.c. time course and altering the shunting resistance.

To prove the problem an electrically equivalent circuit representing the end-plate membrane was used for the end-plate region (Fig. 8A). In Fig. 8A, $E$ is the membrane potential, $R m$ is the membrane resistance and $C$ is the capacity respectively, and $R s$ is a shunting resistance of the membrane. $V_{R}$ is the voltage produced across $R s$, and other symbols are the same as those in Fig. 1. Using the following differential equations, the change of $V_{K}$, the computed e.p.p., was calculated using an analog computor (Fig. 8B).

$$
\begin{aligned}
& E=\left(I_{C}+I_{R}\right) R_{m}+V_{R} \ldots \ldots \ldots \\
& \frac{1}{C} I_{C}=-\frac{d V_{R}}{d t} \quad \ldots \ldots \ldots \cdots \\
& V_{R}=R_{S} \cdot I_{R} \\
& E=R_{m} \cdot C \frac{d V_{R}}{d t}+\left(\frac{R_{m}}{R_{S}}+1\right) V_{R}
\end{aligned}
$$




$$
\frac{d V_{R}}{d t}=-\frac{1}{R_{m} \cdot C}\left(\frac{R_{m}}{R_{S}}+1\right) V_{R}+\frac{E}{R_{m} \cdot C}
$$
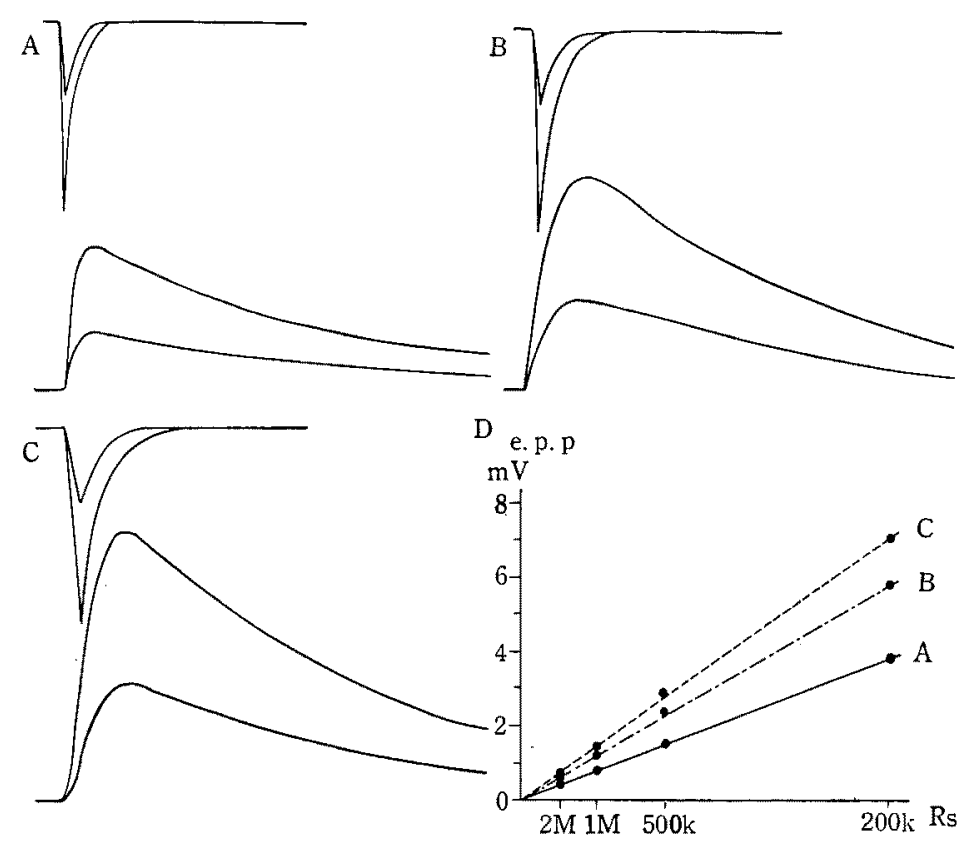

Fig. 9. A, B and C: e.p.c.'s (upper trace) and computed e.p.p.'s (lower curves). In these cases $T$ and $a$ in equation (2) are 0.52 msec and 1.18 in $A, 0.52$ msec and 0.5 in $\mathrm{B}$ and $1.5 \mathrm{msec}$ and 0.5 in C respectively. $R s$ changes from the infinity to $200 \mathrm{~K} \Omega$ in the larger e.p.c. and to $500 \mathrm{~K} \Omega$ in the smaller one. The magnitude of the computed e.p.p.'s becomes larger in order $\mathrm{A}, \mathrm{B}$ and $\mathrm{C}$, i.e., this indicates that the longer the time course of the e.p.c. becomes, the magnitude of the e.p.p. becomes larger. Ordinate in D shows the voltage calibration. D: relationship between the magnitude of the computed e.p.p.'s and the intensity of the e.p.c.'s Line $A, B$, and $C$ indicate the relationship in cases of $A, B$ and $C$ respectively.

Duriug the experiments the value of $R_{S}$ was changed from infinity to certain other values similar to those mentioned in the Results $A$.

Curves in Fig. 9 represent the computed e.p.p.'s obtained by use of the analog computor when values obtained by the experments were substitued for $E, R m$ and $\mathrm{C}$ in the equations. The time course of the computed e.p.p.'s under various conditions was similar to that of the natural e.p.p.'s The values of $T$ and $a$ in the equation (2) were $0.52 \mathrm{msec}$ and 1.18 in the summer at $27^{\circ} \mathrm{C}$ and $1.5 \mathrm{msec}$ and 0.5 in the winter at $15^{\circ} \mathrm{C}$ respectively. As shown in Fig. $9 \mathrm{~A}, \mathrm{~B}$ and $\mathrm{C}$, the rising time to the peak in the computed e.p.p. was much larger than that in the e.p.c. and the magnitude of the former was much smaller than that expected from the 
retio of $R_{m}$ and $R_{S}$, because $T$ and the falling time of the e.p.c. was much shorter than the membrane time constant $\left(R_{m} \times C=20 \mathrm{msec}\right.$. $)$. Furthermore, the amplitude of the computed e.p.p. using the time course of the e.p.c. obtained from the muscle fiber in the winter (Fig. 9C) was larger than that in the summer (Fig. 9A), i.e., the amplitude of the computed e.p.p. depends on the time course of the e.p.c. under the same membrane time constant. When the rate of change of $R_{S}$ was reduced to one third without change in the time course (i.e., the values of $T$ and $a$ in (1) were not altered) the magnitude of the computed e.p.p.'s was altered without changing the time course. It was clear that the times for rising and falling to one-half were almost the same in both the large and the small e.p.p.'s.

From the electrical circuit diagram illustrating the end-plate membrane (Fig. 8A), the reason for the increase of the amplitude of the e.p.p.'sisconsidered is to be one or combination of the following reasons : 1) the increase of $E, 2$ ) the decrease of the minimal value of $R_{S}, 3$ ) prolongation of the time course of $R_{S}$ and 4 ) the increase of $R_{m}$. The increase of the magnitude of the e.p.p. by the action of anticholinesterase is due to the decrease of the minimal value of $R s$ and the prolongation of the time course of $R_{S}$. The increase of the e.p.p. by hyperpolarization of the membrane is due to the increase of $E$. The increase of the e.p.p. in the case of replacement of $\mathrm{Cl}$ ions in Ringer solution with $\mathrm{NO}_{3}$ or $\mathrm{SO}_{4}$ is due to the increase of $R_{m}$.

The effect of $\mathrm{Na}$ ions on the neuromuscular transmission was studied by

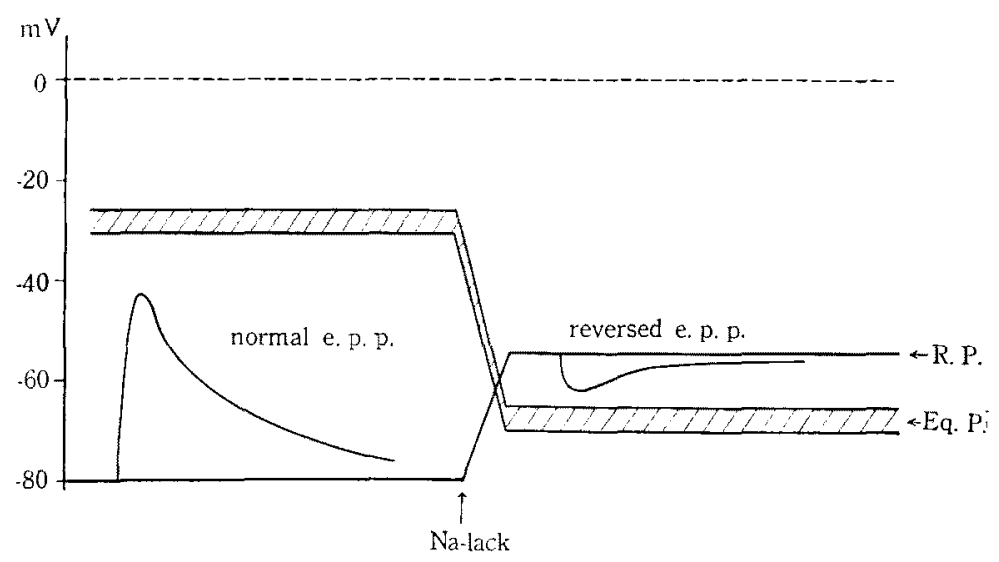

Fig. 10. Schematically summarized diagram of the relation between the resting potential and the equilibrium potential (shaded band) in Ringer and Na-lack sucrose Ringer solution. In Na-lack sucrose Ringer solution the equilibrium potential shifts near to the normal resting potential and the membrane potential goes up over the new equilibrium potential. Thus the e.p.p. reverses in sucrose Ringer solution. 
Fatt \& Katz (1952). They found that lowering the concentration of $\mathrm{Na}$ ions to one-quarter diminished the magnitude of the e.p.p. to less than one-third, and came to the conclusion that there was a close relationship between $\mathrm{Na}$ ions and the release of ACh. Furthermore Castillo \& Katz (1955b) demonstrated the continuous activity of the miniature e.p.p.'s in $\mathrm{K}_{2} \mathrm{SO}_{4}$ solution, and the reversal of ACh potential in sucrose-Ringer solution. It is considered that even in $\mathrm{Na}$ lack solution, $\mathrm{ACh}$ is released from the nerve terminal and reacts with the receptor protein, producing an increase of other ion flux with the exception of $\mathrm{Na}$ ions.

The mechanism for producing the reversed e.p.p. is shown schematically in Fig. 10. By application of Na-lack Ringer solution, the equilibrium potential (indicated by a shaded band) which was -20 to $-30 \mathrm{mV}$ of the membrane potential in normal Ringer solution, shifted near to the normal resting potential level. Also the new resting potential was above the new equilibrium potential. Thus the e.p.p. in normal Ringer solution was reversed in Na-lack solution as shown in the figure. The direction of the e.p.p. might vary depending upon whether the resting potential of the particular fibers was above or below the equilibrium potential.

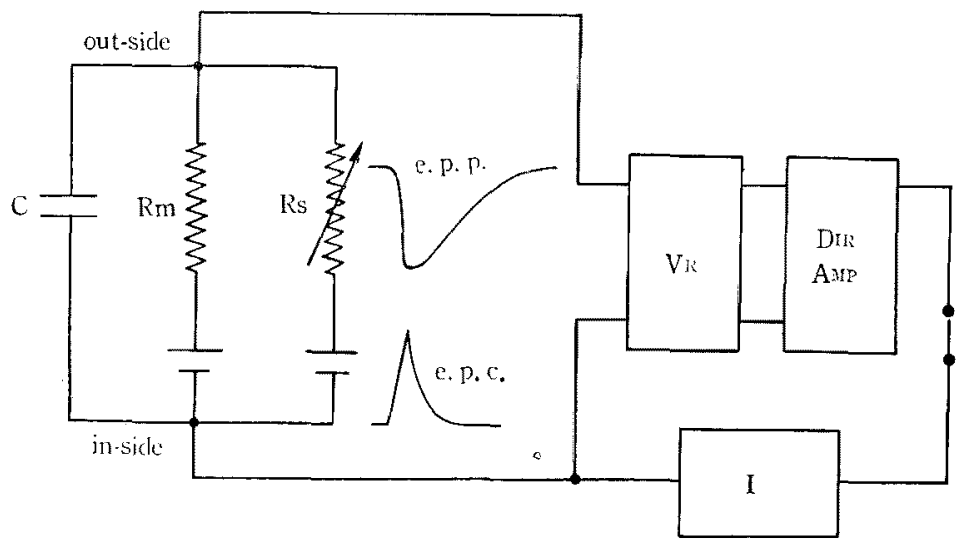

Fig. 11. Schematic diagram of the production of the reversed e.p.p. and e.p.c. Right hand circuit is for the voltage-clamp experiment as shown in Fig. 1 . Symbols as in Fig. 8A. The larger equilibrium potential than the resting potential, is put in series with $R s$. The reversed e.p.p. is quite analogous to the inhibitory postsynaptic potential of other tissues, thus the reversed e.p.c. may be also analogous to the inhibitory synaptic current.

The usual mechanism for producing the e.p.p. by using a shunting resistance across the membrane potential cannot be simply applied for the reversed e.p.p. and the reversed e.p.c. An equilibrium potential larger than the resting potential, as shown in Fig. 11, should be put in series with Rs. The reversed e.p.p. is analogous to the inhibitory postsynaptic potential of the spinal motoneuron or 
other tissue (Brock, Coombs \& Eccles, 1952: Fatt \& Katz 1953: Kuffler \& Eyzaguirre, 1955; Eccles, 1957). Thus the reversed e.p.c. under the voltageclamp condition also might be analogous to the inhibitory synaptic current if it were recorded.

\section{SUMMARY}

1) The property of the motor end-plate was studied by recording the endplate potential (e.p.p.) and the end-plate current (e.p.c.) by means of the intracellular microelectrode method and by measuring the equilibrium potential for the e.p.p. in Na-lack and Cl-lack (Cl ions are replaced with $\mathrm{NO}_{3}, \mathrm{SO}_{4}$ and $\mathrm{I}$ ions) Ringer solution. The e.p.c. measured by the voltage-clamp method in the end-plate region is the current required to keep the membrane potential at the resting level during the neuromuscular transmission and represents the temporal change of the shunting resistance across the membrane potential.

2) The time course of the e.p.c. when compared with that of the e.p.p. showed a characteristic difference. Experiments were made to prove whether a potential similar to the e.p.p. can be reconstructed at the membrane if the shunting resistance is changed according to the time course of the e.p.c. The calculations were made using an analog computor on an electrically equivalent circuit of the end-plate region. The time course of the computed e.p.p.'s under various conditions were similar to that of the natural e.p.p.'s. The relationship between the magnitude of the e.p.p. and the intensity of the e.p.c., and the time course between them under various curarine concentration were discussed.

3) When the membrane potentials under the clamped condition were shifted at various levels for $200 \mathrm{msec}$ and indirect stimulations were applied the relationships between the membrane currents at the e.p.c.'s were obtained. The effective resistance of the resting membrane calculated from those relationship was $200-600 \mathrm{~K} \Omega$. A straight line connecting the peak amplitude of the e.p.c.'s plotted against various clamped voltages of the membrane crossed the abscissa at 50 to $80 \mathrm{mV}$, while the resting potential was usually -70 to $-90 \mathrm{mV}$. Thus the equilibrium potential for the e.p.p. was -10 to $-30 \mathrm{mV}$ of the membrane potential, and the minimum value of the shunting resistance across the membrane potential in the end-plate region, calculated from the tangents formed by these relationships, was $600 \mathrm{~K} \Omega$ to $2 \mathrm{M} \Omega$ under minimum curarized conditions.

4) When the external $\mathrm{Na}$ ions were replaced by sucrose-Ringer solution, the e.p.p. and the e.p.c. were sometimes reversed. The equiliblium potential in Na-lack solution shifted near to the normal resting potential and the resting potential went up towards zero by 20 to $30 \mathrm{mV}$. The mechanism for the production of the reversed e.p.p. and the reversed e.p.c. were discussed from these two potential levels, and it was concluded that the same mechanism produces 
the inhibitory synaptic potential obitaned from other tissues.

5) The effects of various anions, such as $\mathrm{NO}_{3}, \mathrm{SO}_{4}$ and $\mathrm{I}$, on the e.p.p. and the e.p.c. were investigated. The resistance of the resting membrane was increased by about twice by full replacement of Ringer solution with Cl-lack solution. The amplitude of the e.p.p. was enlarged by $\mathrm{NO}_{3}$ but not by $\mathrm{SO}_{4}$.

From the relationships between the magnitude of the e.p.p.'s and the intensity of e.p.c.'s in sucrose and $\mathrm{NO}_{3}$ solution, it was clear that the amplitude of e.p.p. in $\mathrm{NO}_{3}$ increased without an increase of the intensity of the e.p.c. Thus the increase of the e.p.p. in $\mathrm{NO}_{3}$ solution is due to the resistance increase of the resting membrane.

The authors wish to thank Prof. N. Toida for his criticism on this study and reading of this paper and $\mathrm{Mr}$. N. Yoshida of the Department of Communication Engineering, Faculty of Engineering, Kyushu University, for the calculations by the analog computor. This work was supported by a grant from the Ministry of Education and from the Rockefeller Foundation (GA BMR 5748).

\section{References}

1) Brock, L.G., Coombs, J.S. \& Eccles, J.C., Proc. Roy. Soc. B, 1952, 140, 170.

2) Del Castillo, J. \& Katz, B., J. Physiol. 1954a, 124, 560.

3) Del Castillo, J. \& Katz, B., J. Physiol. 1954b, 125, 546.

4) Del Castillo, J. \& Katz, B., J. Physiol. 1955a, 128, 157.

5) Del Castillo, J. \& Katz, B., J. Physiol. 1955b, 128, 396.

6) Eccles, J.C., The Physiology of Nerve Cells. 1957, P. 97, Baltimore : Johns Hopkins Press.

7) Eccles, J.C., Katz, B. \& Kuffler, S.W., J. Neurophysiol. 1942, 5, 211.

8) Eccles, J.C. \& MacFarlane, W.V., J. Neurophysiol. 1949, 12, 59.

9) Edwards, C., Harris, E. J. \& Nishie, K., J. Physiol. 1957, 135, 560.

10) Fatt, P. \& Katz, B., J. Physiol. 1951, 115, 320.

11) Fatt, P. \& Katz, B., J. Physiol. 1952, 117, 109.

12) Fatt, P. \& Katz, B., J. Physiol. 1953, 121, 374.

13) Hagiwara, S. \& Tasalki, I., J. Physiol. 1958, 143, 114.

14) Harris, E.J., J. Physiol. 1958, 141, 351.

15) Harris, E.J. \& Martins-Ferreira, H., J. exp. Biol. 1955, 32, 539.

16) Hill, A.V. \& Macpherson, L., Proc. Roy. Soc. B, 1954, 143, 81.

17) Hodgkin, A.L., Huxley, A.F. \& Katz, B., J. Physiol. 1952, 116, 424.

18) Hodgkin, A.L. \& Horowicz, P., J. Physiol. 1959,148, 127.

19) Hutter, O.F. \& Padsha, S.M., J. Physiol. 1959, 146, 117.

20) Kahn, A.J. \& Sandow, A., Science, 1950, 112, 647.

21) Katz, B., Proc. Roy. Soc. B, 1948, 135, 506.

22) Kuffler, S.W. \& Eyzaguirre, C., J. gen. Physiol. 1955, 39, 155.

23) Ling, G. \& Gerard, R.W., J. cell. comp. Physiol. 1949, 34, 383.

24) Lubin, M., J. cell. comp. Physiol. 1957, 49, 335.

25) Oomura, Y. \& Tomita, T., J. Physiol. Soc. Japan, 1958, 20, 646.

26) Padsha, S.M., J. Physiol. 1957, 137, P 26.

27) Ritchie, J. M., J. Physiol. 1954, 126, 155. 
28) Simon, S.E., Shaw, F.H., Bennett, S. \& Muller, M., J. gen. Physiol. 1957, 40, 753.

29) Takeuchi, N. \& Takeuchi, A., Nature, Lond. 1958, 181, 779.

30) Takeuchi, A. \& Takeuchi, N., J. Neurophysiol. 1959, 22, 395.

31) Tasaki, I. \& Hagiwara, S., Fed. Proc. 1957, 16, 127. 